\title{
Competition of inelastic electron scattering and Interatomic Coulombic Decay in Ne clusters
}

\author{
M. Mucke ${ }^{\mathrm{a}}$, T. Arion ${ }^{\mathrm{b}, \mathrm{c}}$, M. Förstel ${ }^{\mathrm{b}, 1}$, T. Lischke ${ }^{\mathrm{b}, 2}$, U. Hergenhahn $^{\mathrm{d}}$ \\ ${ }^{a}$ Department of Physics and Astronomy, Uppsala University, Box 516, 75120 Uppsala, \\ Sweden \\ ${ }^{b}$ Max Planck Institute for Plasma Physics, Boltzmannstr. 2, 85748 Garching, Germany \\ ${ }^{c}$ Center for Free-Electron Laser Science/DESY, Notkestraße 85, 22607 Hamburg, \\ Germany \\ ${ }^{d}$ Max Planck Institute for Plasma Physics, Wendelsteinstr. 1, 17491 Greifswald, \\ Germany
}

\begin{abstract}
The creation of slow electrons due to Interatomic Coulombic Decay of 2s vacancies in Ne clusters is quantitatively compared to the slow electron yield by intracluster electron impact ionization. Using electron-electron coincidence spectroscopy, both channels can be separated experimentally. Two cluster size distributions, corresponding to two and five to six filled solvation shells, are probed.
\end{abstract}

Keywords: Interatomic Coulombic Decay, Cluster, Autoionization, Electron Scattering

Email addresses: melanie.mucke@physics.uu.se (M. Mucke), uwe.hergenhahn@ipp.mpg.de (U. Hergenhahn)

${ }^{1}$ Present Address: University of Hawai'i at Manoa, 2545 McCarthy Mall, $96816 \mathrm{HI}$ Honolulu, USA

${ }^{2}$ Present Address: Fritz-Haber-Institut der Max-Planck-Gesellschaft, Faradayweg 4-6, 14195 Berlin, Germany 


\section{Introduction}

In extended systems, autoionization is an important deexcitation mechanism of states involving inner valence holes [1-3]. A free electron of low kinetic energy is created as the outcome of these so-called Interatomic or Intermolecular Coulombic Decays (ICD). Quite generally, the role of low kinetic energy electrons in radiation induced chemistry has been realized in recent years [4]. It has therefore been asked, to which extent ICD is contributing to radiochemical damage [5] and whether it could even be used to control radiation induced electron emission $[6,7]$. On the other hand, any energetic particle in matter also produces low kinetic energy electrons by inelastic scattering of the particle itself, and/or its primary reaction products, such as photoelectrons and (fast) Auger electrons [8]. Eventually, most of the primary energy of an energetic particle that passes condensed matter is converted to kinetic energy of slow electrons [8].

Here, we present a study on the relative importance of slow electron creation by ICD, compared to intracluster inelastic electron scattering. The system we chose to discuss these effects is Ne clusters after 2s (inner valence) photoionization with synchrotron radiation.

In the past, neon clusters and their dynamics after valence photoionization have been investigated intensively. In particular for neon dimers, comprehensive experimental and theoretical studies have clarified the different relaxation channels and electron configurations during Interatomic Coulombic Decay $[9,10]$. Most recently, even time-resolved experiments on two types of dimers became possible $[11,12]$. Less experiments on ICD in larger clusters were performed, though, and most of them used conventional photoelectron 
spectrometers [13], which suffer from a reduced transmission in the low kinetic energy range. Furthermore, the assignment of spectral features due to secondary processes, such as ICD, is only possible in an indirect manner, as the connection of primary with secondary electrons is not recorded.

Electron-electron coincidence spectroscopy allows to unequivocally identify the autoionization spectra of certain excited ionic states created by photoionization $[5,14,15]$. In a recent study, this asset was used to quantify the efficiency of the ICD process [16]. An electron-electron coincidence spectrum of Ne clusters was also used as an illustration of the method in [3]. Here, we give a full account of these experiments. Focussing on coincident pairs of two electrons, it is now possible to consider the contributions of photoelectrons and ICD electrons separately from inelastically scattered electrons and background.

Inelastic intracluster photoelectron scattering in Ne clusters has been discussed earlier, and was shown to lead to the production of satellite lines ('excitonic satellites') that are not present in atomic Ne [17]. The excitonic satellites are produced as a result of a valence excitation by inelastic scattering. Their production ratios rapidly decrease away from the respective production threshold. In this paper we will focus on valence ionization by inelastic electron scattering . As a result, two slow electrons with random energy sharing, but fixed total energy, are produced. In a conventional electron spectrometer, such events can only be seen as a gently sloping, continuous background [18]. 


\section{Experimental}

The bulk of experiments were performed at the TGM 4 beamline of the third generation synchrotron radiation source BESSY II (Helmholtz-Zentrum Berlin, Germany) operating in single bunch mode.

To produce the beam of atomic clusters a source already presented earlier [19] was employed. It is based on supersonic expansion of the gaseous sample through a conical nozzle into the vacuum of an expansion chamber (base pressure $10^{-6} \mathrm{mbar}$, during operation $2 \times 10^{-4}$ to $10^{-3} \mathrm{mbar}$ ). Between the expansion chamber and the main chamber (base pressure $2 \times 10^{-7}$ mbar, during operation $10^{-6}$ mbar range), in which the interaction with the synchrotron radiation takes place, the cluster passes a conical skimmer of $1 \mathrm{~mm}$ open diameter (Beam Dynamics Inc., USA). The apparatus is aligned such that the ionization volume coincides with the interaction region of a magnetic bottle electron spectrometer.

Earlier experiments on water clusters showed that a spectrometer of the magnetic bottle type is well suited for the coincident detection of electrons over a wide kinetic energy range [5] as it provides a good transmission down to very low kinetic energies. A set-up dedicated to the investigation of weakly bound clusters was employed in these measurements. The spectrometer design is based on an adaption of earlier instruments [20] to the conditions of BESSY single-bunch operation. In order to achieve flight times below the BESSY repetition time of $800 \mathrm{~ns}$, we have shortened the drift tube to $60 \mathrm{~cm}$. The spectrometer consists essentially of two parts: a permanent magnet to create an inhomogeneous field at the interaction region and a solenoid creating a weak and homogeneous magnetic field along an electrically field 
free drift tube of $0.6 \mathrm{~m}$ length. The drift tube is terminated with a copper mesh behind which an electron detector (a stack of two MCPs aligned in Chevron geometry) is placed. In order to achieve a better defined volume of high magnetic field strength, the magnet is topped with a cone of soft iron. Furthermore, a mesh is fixed in front of it to allow for electrical shielding and to avoid the influence of any charges accumulating atop of the magnet surface on the electrons in the interaction region. Electrons produced in an ionization event are guided by the inhomogeneous magnetic field $(0.3 \mathrm{~T}$ at the surface of magnet) to the entrance aperture of the field-free drift tube, and further by a homogeneous field of $0.6 \mathrm{mT}$ to the detector. In order to improve the energy resolution a retarding voltage was applied between the magnet and the entrance into the drift tube. The spectrometer has been described in further detail elsewhere [21].

Neon clusters were generated in a supersonic expansion of neon gas through a liquid-helium cooled conical nozzle (smallest diameter $50 \mathrm{~mm}$, half opening angle 15 degree) into the vacuum of the expansion chamber. By applying different stagnation pressures (0.85 and 2.1 bar, respectively) of the gas and by varying the nozzle temperature (between 48 and $54 \mathrm{~K}$ ), jets with different cluster size distributions could be produced. The mean cluster size $\langle N\rangle$ was determined from the expansion parameters by using an empirical relation ('scaling law') due to Hagena [22]. In the measurements presented here, three different sizes of neon clusters were investigated: approx. 630, 65 and 42. We will focus on the former two below.

The clusters were irradiated with synchrotron light of $51.8 \mathrm{eV}$ photon energy. This allows the ionization of the inner and outer valence levels of 
neon, $2 \mathrm{~s}$ and $2 \mathrm{p}$, with corresponding atomic ionization potentials of 48.475 and $21.60 \mathrm{eV}$. This means that photoelectrons as well as slow electrons from relaxation via ICD are emitted. Local autoionization channels (Auger decay) are closed. Due to their different kinetic energies, the electrons travel with different velocities towards the detector. Their times of flight are measured with respect to the BESSY bunch marker signal and are passed via a multihit capable time-to-digital-converter to a conventional PC. Every event is recorded separately (list-mode acquisition). In an off-line data analysis process, events are filtered with respect to the number of electrons detected within a coincidence window amounting to two BESSY single bunch periods (1600 ns), and their flight times are converted to kinetic energies. The minimum double pulse resolution dictated by our electronics is $25 \mathrm{~ns}$, and was at 90 ns for the data shown here.

For comparison, few data from a re-analysis of the measurements presented by some of the authors in [16] are also shown. These were recorded at the undulator beamline UE112-PGM-1. Apart from that, the above discussion applies to those data as well.

For discussion, data usually are converted to intensity vs. kinetic energy or binding energy. It is nevertheless rewarding to inspect the raw data in the time-of-flight vs. time-of-flight (TOF) plane (Fig. 1).

\section{Results}

We would like to briefly discuss the principal features seen in the TOF data of Fig. 1 first. Each pixel in this figure corresponds to the intensity received for electron pairs with given values for the TOF. As events are always 


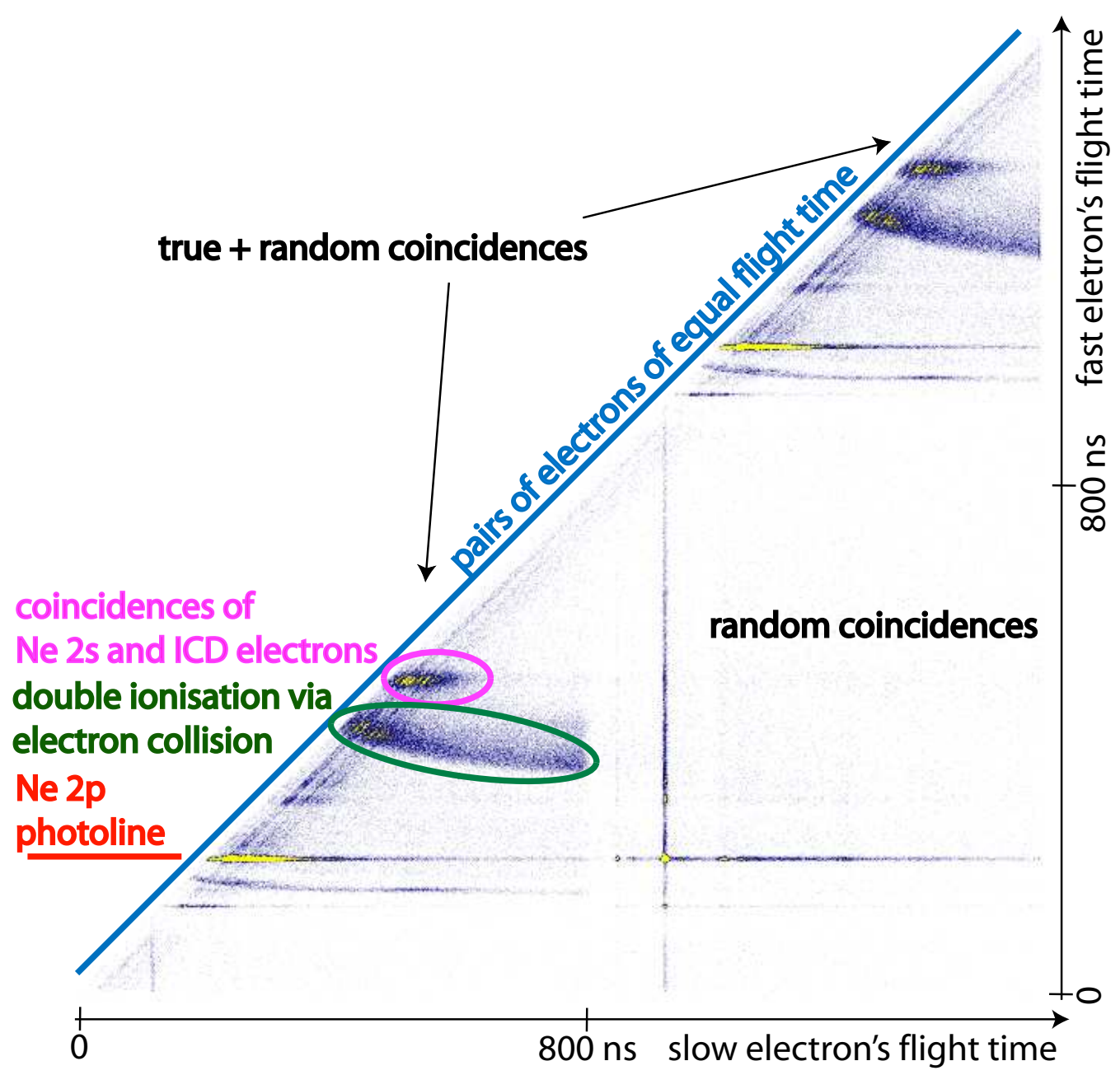

Figure 1: Raw time-of-flight data for electron pairs from Ne clusters at a photon energy of $51.8 \mathrm{eV}$. Intensity is shown on a linear colour scale, vs. times-of-flight $t_{1}$ and $t_{2}$, with $t_{1}<t_{2}$. Data ranging over two full BESSY periods (1600 ns) are shown. This way of acquisition gives a good handle on the separation of true from random coincidence signals. Electron-electron coincidences with both events in the first period (lower left) or both in the second period (upper right) can result from true or random coincidences, events with one electron in the first and the other in the second period (lower right) must be due to a random coincidence. The latter are mostly from two $2 \mathrm{p}$ photoelectrons created by different photons, or a $2 \mathrm{p}$ and an inelastically scatterd electron. Various features due to true coincidence events are discussed in the text. Faint lines following the main diagonal are an artefact produced in the acquisition electronics. 
time-ordered, the sector above the diagonal is unfilled. Below the diagonal, features of different shape in the TOF plane can be seen. This shape depends on the mechanism producing the signal. In photoionization followed by ICD, the energy of the first electron is fixed, the one of the second electron varies somewhat depending on the energy sharing with nuclear degrees of freedom. These events can be seen as horizontal lines, corresponding to fixed TOF of the faster electron. To the contrary, for photo double ionization by inelastic scattering, the energy sharing between the two electrons is arbitrary, and only their total energy is given. The latter is the difference between the photon energy and the final state energy, which amounts to the Coulomb energy which is necessary to produce two vacancies, plus their mutual Coulomb repulsion energy. For the inelastic scattering processes shown here, a $2 \mathrm{p}$ photoelectron produces a second $2 \mathrm{p}^{-1}$ vacancy via intracluster electron impact ionization. Processes of this kind appear at time coordinates given by the implicit function $t_{1}^{-2}+t_{2}^{-2}=$ const., which leads to the bent line at slightly lower $t_{1}$, compared to the ICD feature. These lines become diagonal after conversion of flight times to electron energies. The sector corresponding to $t_{1}$ in the first and $t_{2}$ in the second BESSY period $\left(t_{1}<800 \mathrm{~ns}, t_{2}>800 \mathrm{~ns}\right)$ can only contain random coincidences. This intensity is subtracted from the true+random intensity after time-to-energy conversion. A faint sharp line from direct photo double ionization of the atomic fraction of our gas jet by second diffraction order light is visible at $t_{1}$ coordinates slightly below the random coincidences with the $2 \mathrm{p}$ main line.

The first result of our experiment is shown in Fig. 2. In the two-dimensional plot (bottom right) the number of coincident electron pairs for each combi- 


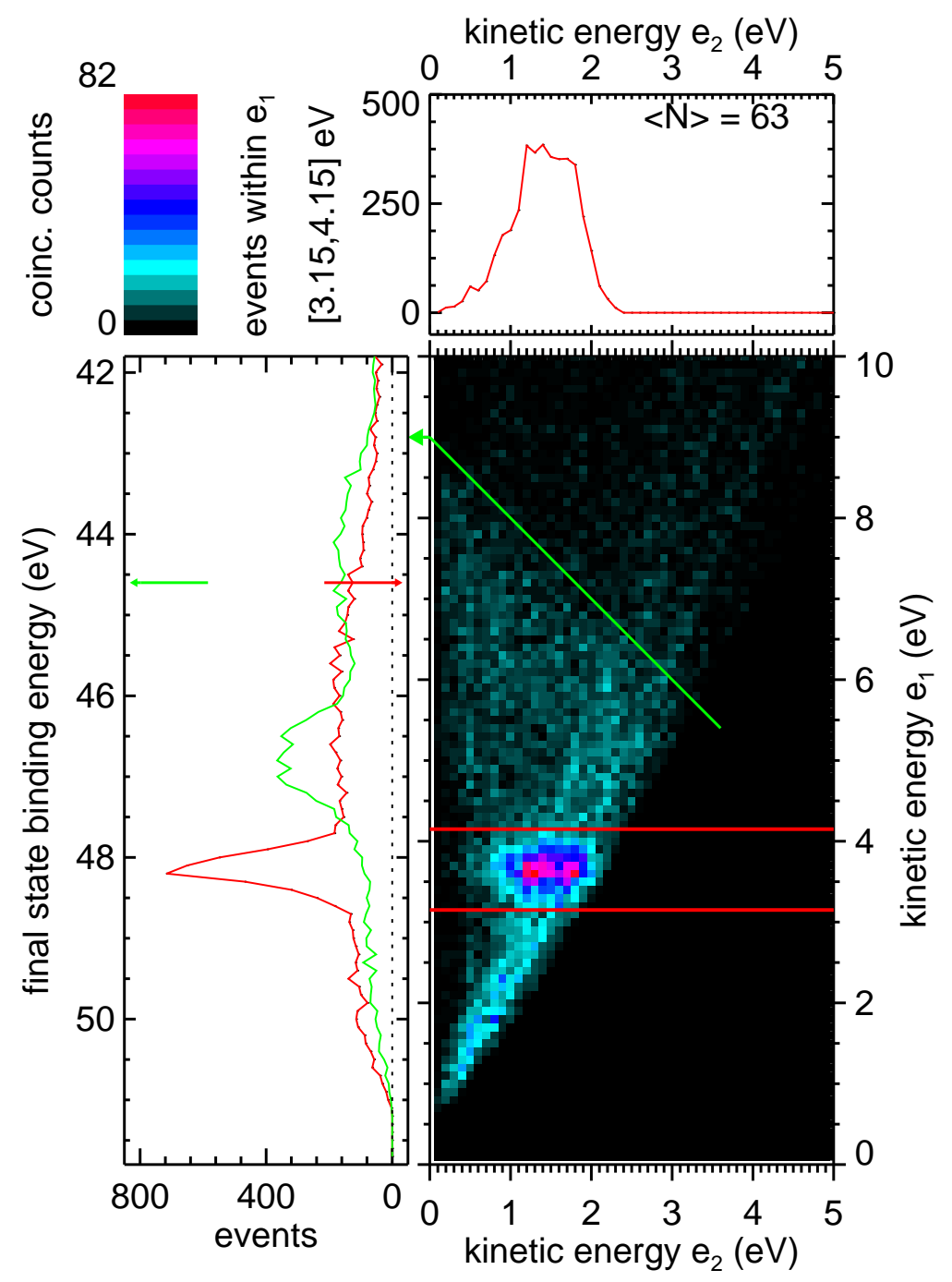

Figure 2: Energy spectrum of electron pairs received from a jet of Ne clusters with $\langle N\rangle=$ 63 , at a photon energy of $51.8 \mathrm{eV}$. Intensity of pairs with a given energy of electron 1 (vertical axis) and electron 2 (horizontal axis) is shown on a linear colour scale. For each pair, $\mathrm{e}_{1}$ denotes the electron of higher kinetic energy. The panel on the left hand side shows the energy spectrum of $\mathrm{e}_{1}$ electrons irrespective of the $\mathrm{e}_{2}$ energy (red trace, which is referred to the energy axis on the right hand side). This corresponds in this graph (approximately) to the photoelectron spectrum. The top panel shows the energy spectrum of $\mathrm{e}_{2}$ electrons, summed up for $\mathrm{e}_{1}$ energies between the two red bars, which designate the binding energy range of the $2 \mathrm{~s}$ inner valence level. The top panel spectrum is the energy spectrum of ICD electrons. Summing up along diagonal lines of constant final state energy (green arrow) gives the green trace in the left hand side panel, referred to the left energy axis (see text for details ). Intensity is expressed as coincident events per pixel $(100 \times 100$ $\left.\mathrm{meV}^{2}\right)$, or coincident events per energy bin $(100 \mathrm{meV})$. 
nation of kinetic energies of the electrons $\left(\mathrm{e}_{1}, \mathrm{e}_{2}\right)$ is shown in a colour coded form (see corresponding intensity scale at top left). The main feature, an intense peak at an $\mathrm{e}_{1}$ energy of $3.1 \mathrm{eV}$ and an $\mathrm{e}_{2}$ energy between 1.0 and $1.8 \mathrm{eV}$, is highlighted by the two red bars. This feature represents electron pairs, in which one of the two electrons $\left(e_{1}\right)$ is a photoelectron emitted from the Ne 2s energy level (binding energy $48.475 \mathrm{eV}$ ) and the other one $\left(\mathrm{e}_{2}\right)$ is of low kinetic energy. Measurements at different photon energies (not shown) confirm that the $e_{2}$ electron is of fixed kinetic energy, independent of the excitation energy. In agreement with earlier experiments [3, 9, 23] and theory [24] we identify these electron pairs with the photoionization of a Ne cluster into a $2 \mathrm{~s}^{-1}$ vacancy state that decays further by ICD. To obtain the energy spectrum of the ICD electron, the intensity of coincident electron pairs with an $e_{1}$ electron in the kinetic energy range of the 2s photoline (marked by the two red bars) was summed up along the $e_{1}$ axis. The resulting spectrum is shown in the top right panel. What becomes obvious both here and in the two dimensional plot is that the energy distribution of the ICD electrons does not extend down to zero eV (see below). Information about the intermediate state can be gathered by performing the summation of electron pairs along the $\mathrm{e}_{2}$ axis for each $\mathrm{e}_{1}$ energy. This results in the red trace given in the left bottom panel, referring to the $e_{1}$ kinetic energy axis on the right hand side of the map. In this diagram, the 2s photoline can be seen at about $3.5 \mathrm{eV}$ of kinetic energy. The bulk-to-surface splitting [25] unfortunately could not be resolved by our spectrometer. An atomic $2 \mathrm{~s}$ feature resulting from uncondensed sample gas is seen in non-coincident spectra from the same experiment (not shown), but not in the figure as it does not lead to emission 
of a second electron.

The mean size assigned to clusters in Fig. 2 is 63. Electron spectra recorded from larger clusters, with $\langle N\rangle=634$, are shown in Fig. 3. Apart from the ICD peak the map contains another prominent feature, namely a relatively broad line along a diagonal that corresponds to a sum of electron kinetic energies of about $8.5 \mathrm{eV}$. This can be understood as a process in which a $2 p$ photoelectron emitted initially ionizes a $2 p$ electron from another monomer in the same cluster (intracluster inelastic scattering). Sharing of the excess energy between the two electrons is arbitrary, which leads to the appearance of a relatively homogeneous diagonal line seen in the spectrum. The total energy (in atomic units) can be estimated as $h \nu-2 * \mathrm{IP}-1 / R$, where $R$ is the distance of the two final state vacancies. Setting for example IP to its atomic value and $R=\infty$, we arrive at $51.8-2 * 21.6=8.6 \mathrm{eV}$, in good agreement with the observation. On the one hand, the neglect of final state Coulomb energy assumes that either the cluster is very large, or the two final state holes are perfectly shielded from each other. On the other hand the cluster IP in fact will be slightly lower than the atomic one. So these two corrections tend to cancel, and we expect our simplest estimate to be a reasonable approximation.

We can obtain additional insight on both processes by summing up the coincident intensity along the lines of constant total electron kinetic energy, as indicated by the green arrow. Doing so one obtains the spectrum shown by the green trace in the lower left panels of Fig. 2 and Fig. 3. This spectrum represents the number of double ionization processes versus (two-hole) final state binding energy. For clarity, the one-dimensional spectra shown in the 


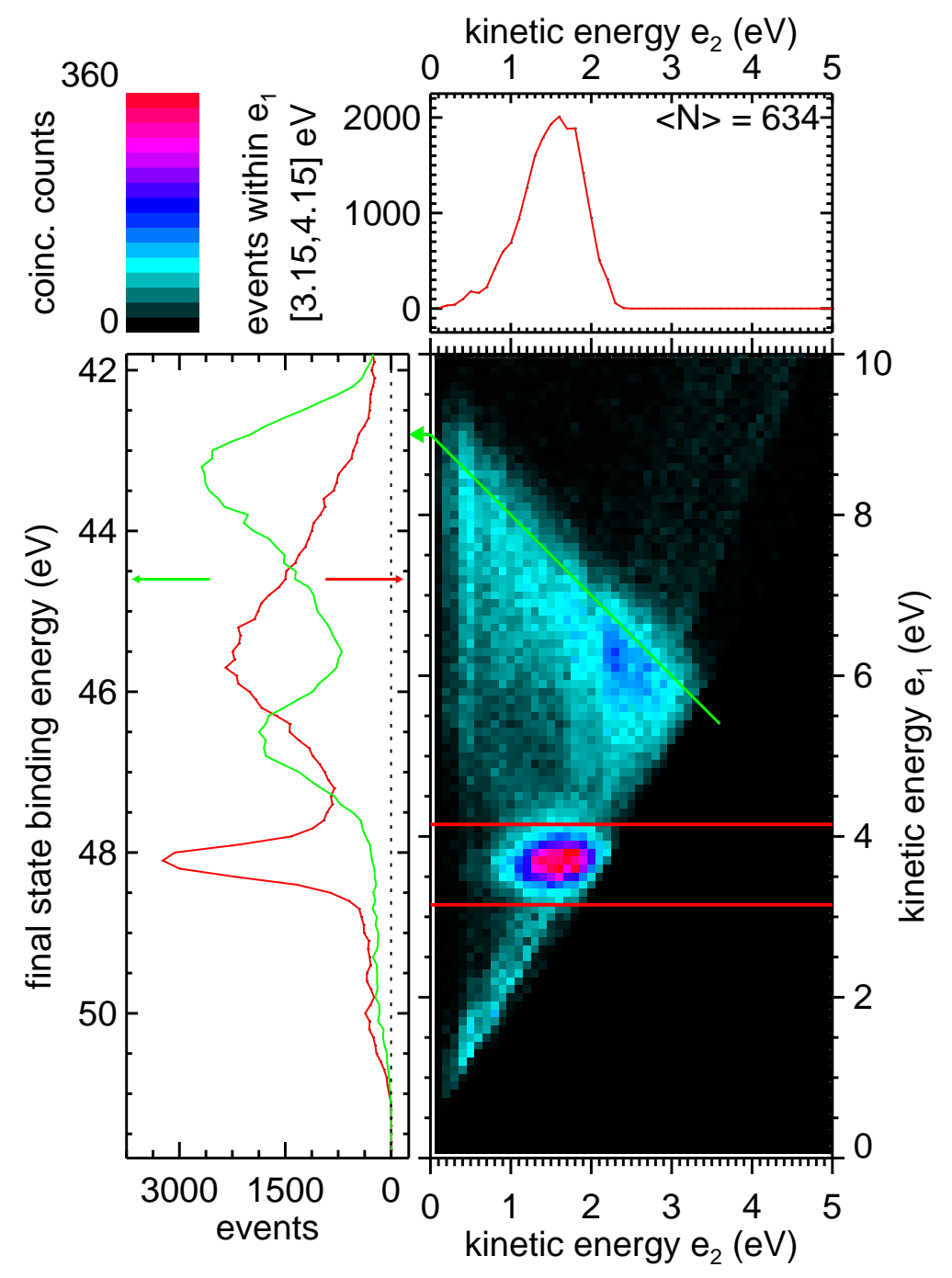

Figure 3: Same as Fig. 2, but for clusters with $\langle N\rangle=634$. 

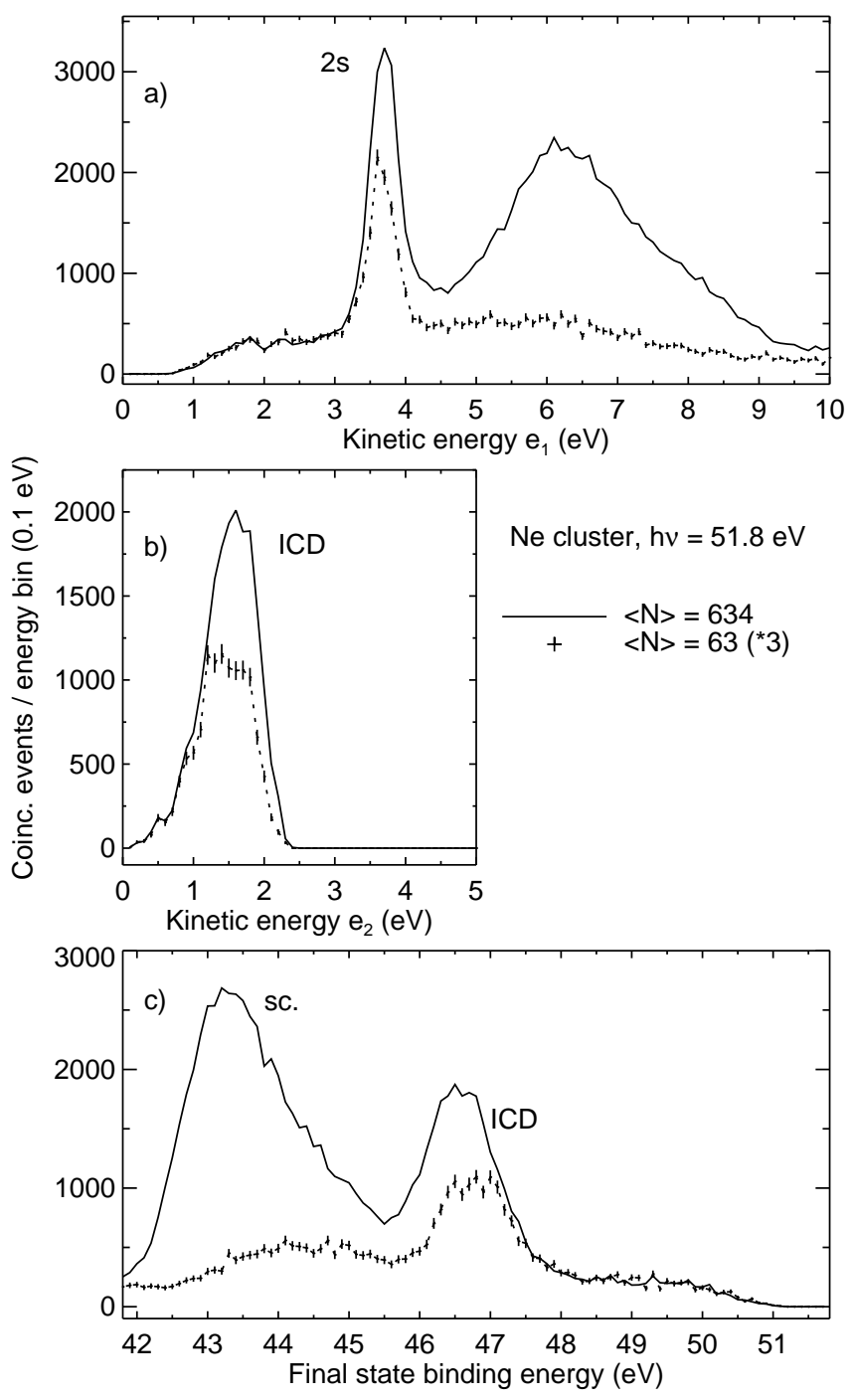

Figure 4: Comparison of the one-dimensional spectra shown in Fig.s 2, 3. Panels a): Kinetic energy of fast electron, b): kinetic energy of slow electron, c): intensity of electron pairs vs. final state energy. Data for smaller clusters (Fig. 2) have been multiplied by a factor of three to ease comparison, and are shown as symbols with statistical error bars. 
combination graphs of Fig. 2 and 3 are compared with each other in Fig. 4. Significant differences are seen in the population of final states shown in panel c). For larger clusters, far more final states corresponding to strongly separated final state holes, and thus lower Coulomb energy, are found. Also, the ICD feature appears to be shifted towards smaller final state energies.

\section{Discussion}

In Fig. 4, for both the photoelectrons and the ICD electrons a small shift towards higher kinetic is visible for the spectra recorded with larger cluster size. A calculation of the respective centers of gravity results in $46 \mathrm{meV}$ shift of the photoline, and $88 \mathrm{meV}$ of the ICD line, at statistical uncertainties of less than $\pm 10 \mathrm{meV}$. The larger the cluster, the more efficient surrounding atoms can shield the site of ionization, so that the Coulombic field the photoelectron has to overcome is lowered, resulting in a higher electron kinetic energy. Interestingly this fact holds as well for the ICD electrons. For ICD, in a larger cluster the binding energies of both initial and final state are lowered by better polarization screening. The effect does not cancel out though, as the screening lowers the energy more effectively in the doubly charged final state than in the singly charged initial state. Thus, the difference energy available for the decay increases, the ICD electron becomes more energetic.

Another explanation for the energy shift of the ICD line would be an increase of contributions from decays involving neighbours in the second coordination shell. While originally ICD was thought to take place to atoms or molecules in the immediate neighbourhood of the initial vacancy only, some of the authors have recently presented evidence for decays involving the sec- 
ond coordination shell of Ne $2 \mathrm{~s}$ vacancies in mixed NeAr clusters [26]. In this example however, the respective energy shift clearly exceeded the one we observe now.

We also note that both ICD peaks do not extend towards zero eV on the kinetic energy axis, which is in contrast to experiments on dimers [9, 24]. Earlier experiments with an electrostatic spectrometer, which had a cut-off for low-energy electrons around $0.4 \mathrm{eV}[13,23]$, are corroborated by that. We attribute this to the nuclear dynamics during the lifetime of the $2 \mathrm{~s}^{-1}$ state: In a dimer, after ionization the nuclear wavepacket travels towards smaller $R$, as the equilibrium state of the Ne dimer cation is more contracted than the neutral. After the decay, thus more energy ends up in the kinetic energy release (KER) of the nuclei, and less is available for the electron energy. In larger rare gas clusters, ionization also leads to a contraction of the bonds around the ionized center, but there is evidence that larger oligomers are formed due to this, e.g. a 13 atom complex consisting of the singly charged center surounded by one full, icosaedric shell of neutral neighbours[27]. For ionized $\mathrm{Ne}_{9}$, this is shown in great detail by a theoretical (molecular dynamics) study [28]. We speculate that due to these differences the $R$ between the two atoms involved in ICD is never becoming as small as in free dimers.

When comparing Fig. 2 to Fig. 3 it becomes obvious that the size of the clusters influences not only the electron kinetic energies but also the extent to which other processes, such as scattering, take place. For the larger clusters (Fig. 3) the diagonal in the coincidence plane is very prominent and, as mentioned above, is centered around a sum of electron kinetic energies of 
$8.5 \mathrm{eV}$. For the smaller clusters (Fig. 2) the feature becomes much weaker. This can be explained by the fact that in a smaller cluster the photoelectrons emitted at or towards the centre of the cluster do not have to pass as many layers of atoms as in the case of a larger cluster, which considerably reduces the scattering probability. Furthermore, the scattering feature shifts to slightly lower energies (8.15 total kinetic eV) for the smaller cluster size. This can be explained by the - on average-lower distance between the two vacancies created by photoionization followed by intracluster scattering in smaller clusters, which leads to a higher Coulomb repulsion energy in the final state. The fact that we see an energy shift of the scattering feature between larger and smaller clusters also allows to discard intercluster inelastic scattering [29] as an alternative explanation for the diagonal feature. If the two electrons were produced from different clusters, the final state spectrum would be independent of cluster size.

One more aspect of the final state energies populated by electron scattering can be seen in Fig. 4c. Here, the difference in energy between the ICD feature and the max. of the scattering peaks amounts to approx. $4 \mathrm{eV}$. This difference is produced by the Coulomb repulsion in the final state. We can approximate its value for ICD to nearest neighbour states, in a distance of $3.4 \AA$ (value for the Ne dimer, [30]). The Coulomb energy corresponding to this distance is about $4.1 \mathrm{eV}$. That is saying that Coulomb repulsion energy is practically vanishing for the least energetic two-hole final states in the larger cluster set. In size, these clusters have between five and six shells, corresponding to $N=561$ and 923 if they are arranged as Mackay icosahedra. To estimate the hole-hole repulsion from that, one would have 
to average over all possible emitter and scatterer sites in the cluster. But even for the four-fold hole-hole distance of the dimer, the repulsion would still correspond to $0.8 \mathrm{eV}$. We therefore conclude that repulsion energies in the two-hole final states are further lowered by shielding of the vacancies, in particular for larger separations.

Our data allow to discuss the locally confined character of slow electron creation by ICD [6]. More precisely, we would like to answer which fraction of slow electrons that are created in the vincinity of an original ionization site results from ICD. Analyzing the respective areas in Fig. 4 shows that the ICD fraction is at 0.59(5) for slow electrons arising within the first and second coordination shell $(\langle N\rangle=63)$ and still at $0.31(5)$ within five to six coordination shells. (We assume that all slow electrons within this range are created by double ionization. In principle, a slow electron can also result from inelastic collisions of a photoelectron below the threshold for creation of another free electron. More than one collision would be needed for that though, see energies given in [17], which we believe is unprobable over the considered range.)

\section{Outlook}

The modelling of elastic and inelastic scattering effects experienced by electrons passing through matter is important in various fields, e.g. radiation chemistry [8], and photoemission of liquids [31]. Our experiments allow to investigate scattering and secondary electron emission from small aggregates of matter, so to say little pieces of the infinitely extended bulk. Scattering effects over various ranges in the material can be probed by varying the 


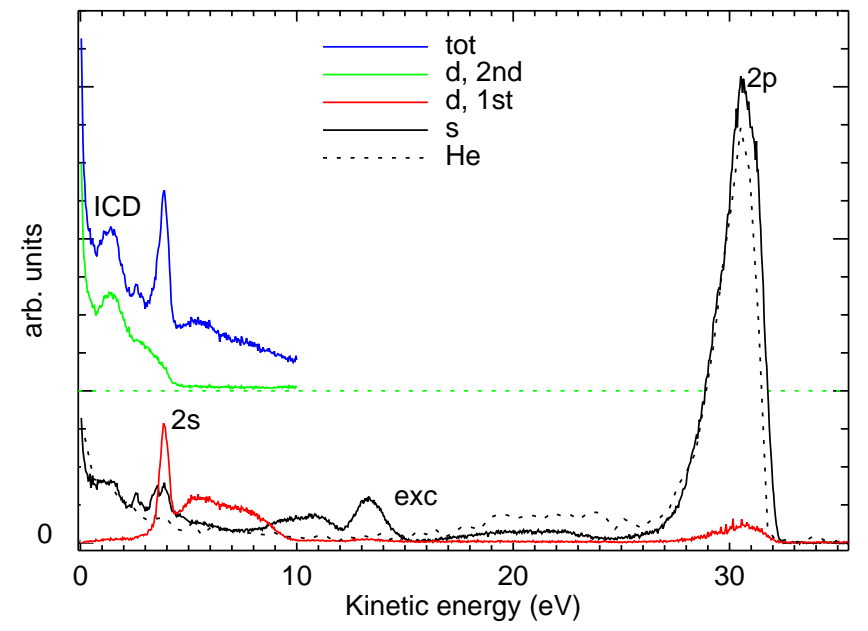

Figure 5: Figure 5: Electron spectra from $\langle N\rangle=480 \mathrm{Ne}$ clusters at $h \nu=52 \mathrm{eV}$, corrected for spectrometer transmission and detection efficiency. Labels refer to 's': single electron emission, 'd, 1st': double photoemission, fast electron (red), 'd, 2nd': double photoemission, slow electron (green), 'tot': sum of all contributions (blue). The two latter are horizontally displaced to increase readability. Features marked are the $2 \mathrm{p}$ and $2 \mathrm{~s}$ photoelectron main lines, the first excitonic satellite and secondary electrons due to ICD. A photoelectron spectrum of gaseous He, scaled to equal height for comparison, is also shown ('He', dotted line). 
clusters size. Figure 5 illustrates the type of data that can be expected. Here, we plot the full electron spectrum of $\langle N\rangle=480$ Ne clusters excited at $h \nu=52 \mathrm{eV}$, and its de-composition into single and double photoemission.

In order to arrive at meaningful data for Fig. 5, a correction for the detection efficiency of the spectrometer is necessary, as detailed in the appendix. No calibration data for this quantity were available for the data set shown in Fig.s 2-4. We have therefore used a re-analysis of the data set presented in [16], to which we had access. (The experimental set-up was similar to the one used above. The reader is referred to the original publication for details.)

In Fig. 5, the production of slow electrons due to $2 \mathrm{~s}$ ionization followed by ICD, and due to inelastic scattering can be seen. A point not discussed yet is the signal from single photoemission. At kinetic energies above 8 $\mathrm{eV}$, it results from the $2 \mathrm{p}$ main line, and its excitonic satellites. Somewhat surprisingly though, even below $8 \mathrm{eV}$ this signal does not drop to zero. Some of that intensity is explicable from contributions of uncondensed Ne atoms in the jet, and from correlation satellites, which might populate this binding energy range [32]. We suggest that most of the intensity observed in the single emission in this kinetic energy region has an origin in apparatus effects though, like electrons losing energy by scattering on spectrometer parts. This can be made plausible by overlaying a photoelectron spectrum of gaseous $\mathrm{He}$ recorded at a similar photon energy (dotted line in Fig. 5). In He, the only physically expected feature is the 1s line. However, again we see a contribution of low kinetic energy electrons which almost equals the one seen in Ne. The amount of higher order radiation in this experiment was below $2 \%$, and cannot explain the observed signals. Although a deeper 
investigation of this topic is certainly warranted, we think that this justifies our above statement that emission of low kinetic energy electrons in the $\mathrm{Ne}$ systems under investigation results mostly from double photoemission, partly by ICD and partly by inelastic scattering. Finally, we note that contributions from apparatus effects in the double photoemission signal would be present with equal extent also in the random coincidence sector, which has been subtracted from the data shown here. Presence of apparatus scattering effects in the double emission data is therefore not expected.

In summary, we have quantitatively decomposed emission of low kinetic energy electrons from Ne clusters into contributions from ICD vs. those from inelastic intracluster scattering. Comparison of data from two different size distributions shows the increasing importance of scattering effects when a more extended aggregate is considered. This type of experiments can be easily extended to systems containing water and organic molecules, which may yield important input data for modelling of electron tracks.

\section{Acknowledgements}

Funding by the Deutsche Forschungsgemeinschaft via the Forschergruppe 1789 is gratefully acknowledged. We thank HZB for the allocation of synchrotron radiation beamtime. 


\section{Appendix A. Transmission correction}

Here we collect the expressions used for correction of analyzer effects in Fig. 5. In the case of single photoemission, the observed spectrum $a_{s}$ as a function of kinetic energy $\varepsilon$ can be written as

$$
a_{s}\left(\varepsilon_{s}\right)=f\left(\varepsilon_{s}\right) p\left(\varepsilon_{s}\right)
$$

where $f$ contains the information on photon flux, target density and the matrix elements for photon-cluster coupling, and $p$ contains all apparatus effects that influence the electron detection ('transmission function'). Analogously, the observed coincidence spectrum for emission of two electrons with energies $\varepsilon_{1}, \varepsilon_{2} ; \varepsilon_{1}>\varepsilon_{2}$ in a double photoionization process can be written as

$$
\tilde{a}\left(\varepsilon_{d, 1}, \varepsilon_{d, 2}\right)=g\left(\varepsilon_{d, 1}, \varepsilon_{d, 2}\right) p\left(\varepsilon_{d, 1}\right) p\left(\varepsilon_{d, 2}\right) .
$$

We can also write expressions for the observed intensity of first electrons, irrespective of the energy of the second electron, and vice versa:

$a_{d, 1}\left(\varepsilon_{d, 1}\right)=p\left(\varepsilon_{d, 1}\right) \int_{0}^{\varepsilon_{d, 1}} d \varepsilon g\left(\varepsilon_{d, 1}, \varepsilon\right) p(\varepsilon) \quad a_{d, 2}\left(\varepsilon_{d, 2}\right)=p\left(\varepsilon_{d, 2}\right) \int_{\varepsilon_{d, 2}}^{h \nu-\varepsilon_{d, 2}} d \varepsilon g\left(\varepsilon, \varepsilon_{d, 2}\right) p(\varepsilon)$.

Instead of the latter two functions, we would like to determine the transmission corrected intensity function for first and second electrons from photo double ionization:

$$
\bar{g}\left(\varepsilon_{d, 1}\right):=\int_{0}^{\varepsilon_{d, 1}} d \varepsilon g\left(\varepsilon_{d, 1}, \varepsilon\right) \text { and } \quad \underline{g}\left(\varepsilon_{d, 2}\right):=\int_{\varepsilon_{d, 2}}^{h \nu-\varepsilon_{d, 2}} d \varepsilon g\left(\varepsilon, \varepsilon_{d, 2}\right) .
$$

These can be determined from the measured data by inserting the relation between $g$ and the observed $\tilde{a}$, yielding e.g. (red trace in Fig. 5)

$$
\bar{g}\left(\varepsilon_{d, 1}\right):=\int_{0}^{\varepsilon_{d, 1}} d \varepsilon \frac{\tilde{a}\left(\varepsilon_{d, 1}, \varepsilon\right)}{p\left(\varepsilon_{d, 1}\right) p(\varepsilon)} .
$$


Table B.1: The expansion conditions used to produce the clusters are given here in tabular form. Nozzle parameters: Conical, half opening angle $15^{\circ}$. Estimates of $\langle N\rangle$ are according to $[22]$.

\begin{tabular}{ccccc}
\hline \hline Fig. & $\begin{array}{c}\text { nozzle diameter } \\
(\mu \mathrm{m})\end{array}$ & $\begin{array}{c}\text { stagnation pressure } \\
(\text { bar })\end{array}$ & $\begin{array}{c}\text { temperature } \\
\langle N\rangle\end{array}$ \\
\hline 2 & 50 & 0.85 & 50.3 & 64 \\
3 & 50 & 2.1 & 48.7 & 634 \\
\hline 5 & 80 & 1.2 & 48 & 480 \\
\hline
\end{tabular}

The corrected expression for single photoemission taking place in competition with double photoemission is a bit more complicated, as some of the observed single emission intensity $a_{s}$ may result from double emission events, in which one of the electrons has not been detected due to apparatus effects. We have $a_{s}\left(\varepsilon_{s}\right)=f\left(\varepsilon_{s}\right) p\left(\varepsilon_{s}\right)+p\left(\varepsilon_{s}\right) \int_{0}^{\varepsilon_{s}} d \varepsilon g\left(\varepsilon_{s}, \varepsilon\right)(1-p(\varepsilon))+p\left(\varepsilon_{s}\right) \int_{\varepsilon_{s}}^{h \nu-\varepsilon_{s}} d \varepsilon g\left(\varepsilon_{s}, \varepsilon\right)(1-p(\varepsilon))$.

Reorganizing terms and inserting above results we finally arrive at (black trace in Fig. 5)

$f\left(\varepsilon_{s}\right)=\frac{1}{p\left(\varepsilon_{s}\right)} \cdot\left[a_{s}\left(\varepsilon_{s}\right)-\int_{0}^{\varepsilon_{s}} d \varepsilon \tilde{a}\left(\varepsilon_{s}, \varepsilon\right) \frac{1-p(\varepsilon)}{p(\varepsilon)}-\int_{\varepsilon_{s}}^{h \nu-\varepsilon_{s}} d \varepsilon \tilde{a}\left(\varepsilon, \varepsilon_{s}\right) \frac{1-p(\varepsilon)}{p(\varepsilon)}\right]$.

\section{Appendix B. Expansion conditions}

See Tab. B.1. 


\section{References}

[1] L. S. Cederbaum, J. Zobeley, F. Tarantelli, Giant intermolecular decay and fragmentation of clusters, Phys. Rev. Lett. 79 (1997) 4778-4781.

[2] V. Averbukh, P. Demekhin, P. Kolorenc, S. Scheit, S. D. Stoychev, A. I. Kuleff, Y.-C. Chiang, K. Gokhberg, S. Kopelke, N. Sisourat, L. S. Cederbaum, Interatomic electronic decay processes in singly and multiply ionized clusters, J. Electron Spectrosc. Relat. Phenom. 183 (1-3) (2011) 36 .

[3] U. Hergenhahn, Interatomic and intermolecular coulombic decay: The early years, J. Electron Spectrosc. Relat. Phenom. 184 (3-6) (2011) 78. URL $10.1016 / j$.elspec. 2010.12.020

[4] E. Alizadeh, L. Sanche, Precursors of solvated electrons in radiobiological physics and chemistry., Chem. Rev. 112 (11) (2012) 5578. doi:10.1021/cr300063r.

[5] M. Mucke, M. Braune, S. Barth, M. Förstel, T. Lischke, V. Ulrich, T. Arion, U. Becker, A. Bradshaw, U. Hergenhahn, A hitherto unrecognized source of low-energy electrons in water, Nature Physics 6 (2010) $143-146$.

[6] K. Gokhberg, P. Kolorenc, A. I. Kuleff, L. S. Cederbaum, Site- and energy-selective slow-electron production through intermolecular coulombic decay., Nature $505 \quad$ (7485) (2014) 661. doi:10.1038/nature12936. 
[7] F. Trinter, M. S. Schöffler, H.-K. Kim, F. P. Sturm, K. Cole, N. Neumann, A. Vredenborg, J. Williams, I. Bocharova, R. Guillemin, M. Simon, A. Belkacem, A. L. Landers, T. Weber, H. Schmidt-Böcking, R. Dörner, T. Jahnke, Resonant auger decay driving intermolecular coulombic decay in molecular dimers., Nature 505 (7485) (2014) 664. doi:10.1038/nature12927.

[8] S. M. Pimblott, J. A. LaVerne, Production of low-energy electrons by ionizing radiation, Radiat. Phys. Chem. 76 (8-9) (2007) 1244.

[9] T. Jahnke, A. Czasch, M. S. Schöffler, S. Schössler, A. Knapp, M. Käsz, J. Titze, C. Wimmer, K. Kreidi, R. E. Grisenti, A. Staudte, O. Jagutzki, U. Hergenhahn, H. Schmidt-Böcking, R. Dörner, Experimental observation of interatomic coulombic decay in neon dimers, Phys. Rev. Lett. 93 (2004) 163401.

[10] K. Kreidi, T. Jahnke, T. Weber, T. Havermeier, X. Liu, Y. Morisita, S. Schössler, L. P. H. Schmidt, M. Schöffler, M. Odenweller, N. Neumann, L. Foucar, J. Titze, B. Ulrich, F. Sturm, C. Stuck, R. Wallauer, S. Voss, I. Lauter, H. K. Kim, M. Rudloff, H. Fukuzawa, G. Prümper, N. Saito, K. Ueda, A. Czasch, O. Jagutzki, H. Schmidt-Böcking, S. Stoychev, P. V. Demekhin, R. Dörner, Relaxation processes following $1 s$ photoionization and auger decay in $n e_{2}$, Phys. Rev. A 78 (4) (2008) 043422. doi:10.1103/PhysRevA.78.043422.

[11] K. Schnorr, A. Senftleben, M. Kurka, A. Rudenko, L. Foucar, G. Schmid, A. Broska, T. Pfeifer, K. Meyer, D. Anielski, R. Boll, D. Rolles, M. Kübel, M. F. Kling, Y. H. Jiang, S. Mondal, T. Tachibana, K. Ueda, 
T. Marchenko, M. Simon, G. Brenner, R. Treusch, S. Scheit, V. Averbukh, J. Ullrich, C. D. Schröter, R. Moshammer, Time-resolved measurement of interatomic coulombic decay in ne ${ }_{2}$, Phys. Rev. Lett. 111 (2013) 093402. doi:10.1103/PhysRevLett.111.093402.

URL http://link.aps.org/doi/10.1103/PhysRevLett.111.093402

[12] F. Trinter, J. B. Williams, M. Weller, M. Waitz, M. Pitzer, J. Voigtsberger, C. Schober, G. Kastirke, C. Müller, C. Goihl, P. Burzynski, F. Wiegandt, T. Bauer, R. Wallauer, H. Sann, A. Kalinin, L. P. H. Schmidt, M. Schöffler, N. Sisourat, T. Jahnke, Evolution of interatomic coulombic decay in the time domain, Phys. Rev. Lett. 111 (2013) 093401. doi:10.1103/PhysRevLett.111.093401.

URL http://link.aps.org/doi/10.1103/PhysRevLett.111.093401

[13] S. Barth, S. Marburger, O. Kugeler, V. Ulrich, S. Joshi, A. M. Bradshaw, U. Hergenhahn, The efficiency of interatomic coulombic decay in ne clusters, Chem. Phys. 329 (2006) 246-250.

[14] T. Jahnke, H. Sann, T. Havermeier, K. Kreidi, C. Stuck, M. Meckel, M. Schöffler, N. Neumann, R. Wallauer, S. Voss, A. Czasch, O. Jagutzki, A. Malakzadeh, F. Afaneh, T. Weber, H. Schmidt-Böcking, R. Dörner, Ultrafast energy transfer between water molecules, Nature Physics 6 (2) (2010) 139-142.

[15] T. Arion, O. Takahashi, R. Püttner, V. Ulrich, S. Barth, T. Lischke, A. M. Bradshaw, M. Förstel, U. Hergenhahn, Conformational and nuclear dynamics effects in molecular auger spectra : 
fluorine core-hole decay in cf 4, J. Phys. B 47 (2014) 124033. doi:http://dx.doi.org/10.1088/0953-4075/47/12/124033.

[16] M. Förstel, T. Arion, U. Hergenhahn, Measuring the efficiency of interatomic coulombic decay in ne clusters, J. Electron Spectros. Relat. Phenomena 191 (2013) 16, vUVX Proc. doi:http://dx.doi.org/10.1016/j.elspec.2013.11.002.

[17] U. Hergenhahn, A. Kolmakov, M. Riedler, A. R. B. d. Castro, O. Löfken, T. Möller, Observation of excitonic satellites in the photoelectron spectra of ne and ar clusters, Chem. Phys. Lett. 351 (2002) 235.

[18] R. Wehlitz, F. Heiser, O. Hemmers, B. Langer, A. Menzel, U. Becker, Electron-energy and angular distributions in the double photoionization of helium, Phys. Rev. Lett. 67 (1991) 3764, he2.

[19] S. P. Marburger, O. Kugeler, U. Hergenhahn, A molecular beam source for electron spectroscopy of clusters, AIP Conference Proceedings 705 (1) (2004) 1114-1117. doi:10.1063/1.1757994.

URL http://link.aip.org/link/?APC/705/1114/1

[20] F. Penent, P. Lablanquie, R. I. Hall, J. Palaudoux, K. Ito, Y. Hikosaka, T. Aoto, J. H. D. Eland, Coincidence auger spectroscopy, J. Electron Spectrosc. Relat. Phenomena 144-147 (2005) 7.

[21] M. Mucke, M. Förstel, T. Lischke, T. Arion, A. M. Bradshaw, U. Hergenhahn, Performance of a short magnetic bottle electron spectrometer., Rev. Sci. Instrum. 83 (6) (2012) 063106.

URL http://link.aip.org/link/?RSINAK/83/063106/1 
[22] O. F. Hagena, Cluster ion sources, Rev. Sci. Instrum. 63 (1992) 23742379 .

[23] S. Marburger, O. Kugeler, U. Hergenhahn, T. Möller, Experimental evidence for interatomic coulombic decay in ne clusters, Phys. Rev. Lett. 90 (2003) 203401.

[24] S. Scheit, V. Averbukh, H.-D. Meyer, N. Moiseyev, R. Santra, T. Sommerfeld, J. Zobeley, L. S. Cederbaum, On the interatomic coulombic decay in the ne dimer, J. Chem. Phys. 121 (17) (2004) 8393-8398.

[25] G. Öhrwall, M. Tchaplyguine, M. Lundwall, R. Feifel, H. Bergersen, T. Rander, A. Lindblad, J. Schulz, S. Peredkov, S. Barth, S. Marburger, U. Hergenhahn, S. Svensson, O. Björneholm, Femtosecond interatomic coulombic decay in free neon clusters: Large lifetime differences between surface and bulk, Phys. Rev. Lett. 93 (2004) 173401.

[26] E. Fasshauer, M. Förstel, S. Pallmann, M. Pernpointner, U. Hergenhahn, Using icd for structural analysis of clusters: a case study on near clusters, New J. Phys. 16 (10) (2014) 103026. doi:10.1088/1367$2630 / 16 / 10 / 103026$.

[27] F. Carnovale, J. B. Peel, R. G. Rothwell, J. Valldorf, P. J. Kuntz, Photoelectron spectroscopy of argon clusters: Evicence for an ar 13 ionization chromophore, J. Chem. Phys. 90 (1989) 1452. doi:http://dx.doi.org/10.1063/1.456087.

[28] D. Bonhommeau, A. Viel, N. Halberstadt, Dissociative ionization of 
neon clusters nen $\mathrm{n},=3$ to 14: A realistic multisurface dynamical study, J. Chem. Phys. 123 (5) (2005) 1. doi:10.1063/1.1953530.

[29] A. Knop, H. W. Jochims, A. L. D. Kilcoyne, A. P. Hitchcock, E. Rühl, Zero-kinetic-energy photoelectron spectroscopy of ar(2p)-excited argon clusters, Chem. Phys. Lett. 223 (5-6) (1994) 553.

[30] A. Wüest, F. Merkt, Determination of the interaction potential of the ground electronic state of ne[sub 2] by high-resolution vacuum ultraviolet laser spectroscopy, J. Chem. Phys. 118 (19) (2003) 8807.

[31] S. Thürmer, R. Seidel, M. Faubel, W. Eberhardt, J. C. Hemminger, S. E. Bradforth, B. Winter, Photoelectron angular distributions from liquid water: Effects of electron scattering, Phys. Rev. Lett. 111 (17) (2013) 173005. doi:10.1103/PhysRevLett.111.173005.

[32] P. Bolognesi, L. Avaldi, D. R. Cooper, M. Coreno, R. Camilloni, G. C. King, High-resolution threshold photoelectron measurements of the ne 2p4 nl satellite states, J. Phys. B 35 (2002) 2927. 